\title{
High-speed Coherent Optical Communication with Isolator-free Heterogeneous Si/III-V Lasers
}

\author{
Zhewei Zhang, Kaiheng Zou, Huolei Wang, Peicheng Liao, Naresh Satyan, George Rakuljic, Alan \\ E.Willner, Fellow, IEEE and Amnon Yariv, Life Fellow, IEEE
}

\begin{abstract}
Coherent optical communication is considered as an indispensable solution to the ever-increasing demand for higher data rates. To reduce the cost and form factor of coherent transceivers, full integration of photonic devices including lasers, modulators, amplifiers, photodetectors and other components is necessary. However, as fabricating optical isolators on chip remains extremely challenging, optical feedback, which can degrade the coherence of semiconductor lasers, becomes the main obstacle, thwarting large-scale photonic integration. An appealing solution to such a problem is to use semiconductor lasers with intrinsic insensitivity to optical feedback as the integrated light sources. The heterogenous $\mathrm{Si} / \mathrm{III}-\mathrm{V}$ lasers, with their built-in high-Q resonators, are expected to possess a robustness to optical feedback which exceeds by several orders of magnitude compared to commercial III-V distributed feedback (DFB) lasers, which will be validated here. We present data showing that the heterogeneous $\mathrm{Si} / \mathrm{III}-\mathrm{V}$ lasers can preserve their phase coherence under much larger optical feedback and therefore function without severe degradation in isolator-free coherent optical communication systems.
\end{abstract}

This work is supported by National Science Foundation (Y501119), DARPA MTO (N66001-14-1-4062) and US Army Research Office (W911NF-16-C-0026 and W911NF-14-P-0020). We would like to acknowledge the technical and fabrication infrastructure support from Kavli Nanoscience Institute at California Institute of Technology.

Z. Zhang is with the Department of Applied Physics and Materials Science, California Institute of Technology, Pasadena, CA 91125 USA (e-mail: zzzhang@caltech.edu).

K. Zou is with the Department of Electrical Engineering, University of Southern California, Los Angeles, CA 90089 USA (e-mail: kaihengz@usc.edu).

H. Wang is with the Department of Applied Physics and Materials Science, California Institute of Technology, Pasadena, CA 91125 USA (e-mail: hlwang@caltech.edu).

P. Liao is with the Department of Electrical Engineering, University of Southern California, Los Angeles, CA 90089 USA (e-mail: peicheng@usc.edu).

N. Satyan is with the Telaris Inc., Santa Monica, CA 90403 USA (e-mail: satyan@telarisinc.com).

G. Rakuljic is with the Telaris Inc., Santa Monica, CA 90403 USA (e-mail: rakuljic@telarisinc.com).

A. Willner is with the Department of Electrical Engineering, University of Southern California, Los Angeles, CA 90089 USA (e-mail: willner@usc.edu).

A. Yariv is with the Department of Applied Physics and Materials Science and the Department of Electrical Engineering, California Institute of Technology, Pasadena, CA 91125 USA (e-mail: ayariv@caltech.edu).
Index Terms_-Laser theory, laser noise, laser applications.

\section{INTRODUCTION}

$\mathrm{A}_{\mathrm{t}}^{\mathrm{s}}$ $\mathrm{S}$ the requirement for the data rate handled by a single transceiver exceeds $400 \mathrm{~Gb} / \mathrm{s}$ in the up-coming optical communication networks [1], the deployment of coherent optical communication systems becomes necessary in order to satisfy the ever-increasing demands [2-4]. The main obstacle to the transition is the high cost of coherent transceivers, to which the most likely solution is photonic integration, bringing semiconductor lasers, modulators, amplifiers, photodetectors and other photonic devices together on a single platform. However, as optical isolators cannot be monolithically integrated due to their bulky size and incompatibility with modern CMOS fabrication techniques, optical feedback, from either unwanted reflection or spontaneous emission in optical amplifiers, degrades the coherence of the semiconductor lasers, which can be fatal to the system performance [5].

Conventional III-V DFB lasers, the main light sources in the today's optical communication networks, are quite sensitive to optical feedback. Even a very small faction of optical feedback, can cause dramatic degradation of their performance [6], leading to the fact that an optical isolator must be appended to every III-V DFB laser for practical applications. Hence, for photonic integration, it is straightforward to replace the conventional III-V DFB lasers with monolithic semiconductor lasers with intrinsic insensitivity to optical feedback. The heterogeneous Si/III-V lasers, with their built-in high-Q resonators to block a large amount of optical feedback, are expected to be much more insensitive to optical feedback than III-V DFB lasers, whose quality factors are orders of magnitude lower.

Recently, there have been several papers investigating the feedback sensitivity of heterogeneous Si/III-V lasers in the communication systems and claiming their superb robustness. However, these experiments are not that rigorous as either Fabry-Perot (FP) lasers, i.e. multi-mode lasers, are used in the measurement or there lacks comparison between the Si/III-V lasers and other lasers in their performance [7-10].

In this paper, we are going to examine the feedback sensitivity of the Si/III-V laser and compare it to a conventional III-V DFB laser which is commercially available (QPhotonics QDFBLD-1550-5AX unpackaged with optical isolators). The results illustrate that the Si/III-V lasers can maintain their phase 
coherence under much larger optical feedback and function without severe degradation in the isolator-free coherent optical communication system, rendering them strong contenders to be the integrated light sources.

\section{OPTICAL FEEDBACK}

Optical feedback can be classified into two categories, namely coherent and incoherent optical feedback. Coherent optical feedback, such as the light reflected from an external mirror, is explicitly correlated with the laser output while incoherent optical feedback, like amplified spontaneous emission (ASE), is typically originated from a different and independent light source, in this case an optical amplifier, and uncorrelated with the laser output. What we are going to explain in this section is how coherent and incoherent optical feedback modifies the laser coherence, characterized by the frequency noise power spectral density (PSD), respectively.

In semiconductor lasers, both the intensity noise and the phase noise arise from spontaneous emission, a quantum noise source. Besides, the intensity noise is coupled to the phase noise through the mechanism called linewidth enhancement, characterized by Henry's $\alpha$ alpha parameter [11], as shown in Fig. 1(a). The noise source and the coupling together determine the frequency noise PSD of a semiconductor laser. By introducing optical feedback into the laser system, either the noise source or the coupling between noises becomes different, resulting in the change of the laser frequency noise PSD.

(a)

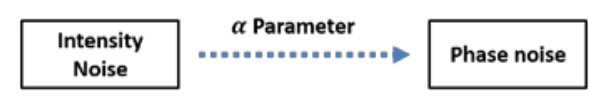

(b)

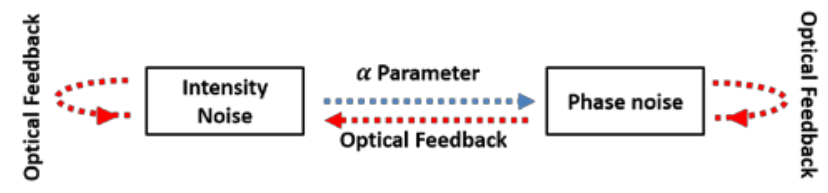

(c)

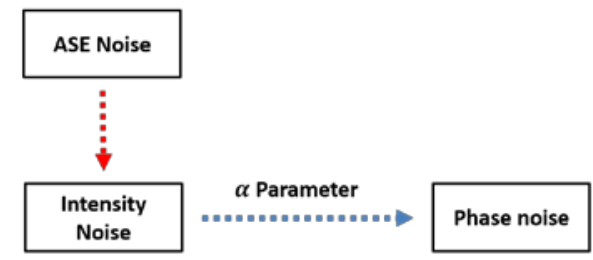

Fig. 1. Noise coupling mechanism of a semiconductor laser (a) under no optical feedback; (b) under coherent optical feedback and (c) under incoherent optical feedback.

In the case of coherent optical feedback, the noise source is still spontaneous emission as external mirrors or equivalent are passive devices. However, due to its correlation to the laser output and thus the laser field inside the resonator, additional coupling channels between the laser intensity and phase noises are created, forming positive feedback loops between noises, as shown in Fig. 1(b). When the feedback becomes strong enough, multiple radio frequency (RF) oscillations, satisfying certain phase conditions, can take place in the laser frequency noise PSD, which has been predicted in the numerical calculation
[12]. Equivalently, based on the Central Relation between laser frequency noise PSD and optical lineshape [13], the original single-mode laser becomes multi-mode, known as coherence collapse [6, 14]. The effects of coherent optical feedback on semiconductor lasers can be quantified by the C-parameter, which was first introduced by Petermann [15]

$$
C=\frac{\tau_{\text {ext }}}{\tau_{\text {laser }}} \frac{r_{\text {ext }}}{r_{\text {laser }}}\left(1-\left|r_{\text {laser }}\right|^{2}\right) \sqrt{1+\alpha^{2}}
$$

, where $\tau_{\text {ext }}$ and $\tau_{\text {laser }}$ are the round trip time of the external and laser cavities, respectively. $r_{\text {ext }}$ and $r_{\text {laser }}$ are the reflectivity of the external and laser mirrors, respectively. Although the C-parameter is originally defined based on a simple model of FP lasers and should be modified when it comes to lasers with more complex structure [16], it does provide us with the correct physical picture of what roles some key parameters, such as the laser mirror reflectivity, the size of the laser cavity and the length of the external optical feedback loop, play in such a type of problems. In Petermann's analysis, laser coherence will be severely affected once the C-parameter exceeds unity. Based on the previous definition, it is impossible to characterize lasers' sensitivity to coherent optical feedback in any absolute sense because the effects depend on not only the feedback level but also the distance between the laser and the external reflection point, which varies from one scenario to another. However, in any given situation, the relative difference between any two lasers' feedback sensitivity is the same as the ratio between the corresponding C-parameters is only dependent on the intrinsic parameters of the lasers

$$
\eta \equiv \frac{C_{1}}{C_{2}}=\frac{\tau_{\text {laser } 2}}{\tau_{\text {laser } 1}} \frac{r_{\text {laser } 2}}{r_{\text {laser } 1}} \frac{1-\left|r_{\text {laser } 1}\right|^{2}}{1-\left|r_{\text {laser } 2}\right|^{2}} \frac{\sqrt{1+\alpha_{1}^{2}}}{\sqrt{1+\alpha_{2}^{2}}}
$$

, where the parameters with the subscripts 1 and 2 belong to laser 1 and 2, respectively. Such a ratio $\eta$ also indicates that the feedback effect on laser 1 will be the same as that on laser 2 if the external mirror reflectivity is changed by a factor of $\eta$ for laser 1 , which is the criterion for our following measurements to characterize lasers' sensitivity to coherent optical feedback.

On the contrary, incoherent optical feedback such as ASE noise, originated from an independent light source, naturally serves as a new noise source. Because it is uncorrelated to the laser output, the additional noise induced in the laser resonator is pure intensity noise, which is eventually coupled to the phase noise through the same mechanism of linewidth enhancement. Hence, the power of the noise injected into the laser resonator solely determines the degree of the laser's coherence degradation. In this case, the feedback effects only depend on the feedback level so that we can characterize the lasers' sensitivity to incoherent optical feedback in an absolute sense.

In the coherent optical communication, either the rising of side modes or the increase of the laser phase noise can be catastrophic to the system performance. To enhance the lasers' robustness, preventing the optical feedback from entering the laser resonator is the key, which can be achieved by employing mirrors with the reflectivity approaching to unity. However, such an approach cannot be applied to conventional III-V DFB 
lasers as it will reduce dramatically the output power because of their large internal loss in laser resonators, rendering the III-V DFB lasers useless. To resolve such a problem, it is necessary for semiconductor lasers to have resonators with very low internal loss so that much stronger optical field can be built inside the resonator to compensate for the reduction of the output coupling in order to get the same output power.

More specifically, the condition of the optimization of laser output power is given by [17]

$$
T_{\text {opt }}=-L_{i}+\sqrt{g_{0} L_{i}}
$$

, where $T_{\text {opt }}$ represents the optimized useful output and $L_{i}$ is the linear internal loss. $g_{0}$ is the unsaturated gain. The heterogeneous Si/III-V lasers are designed in a way that most of the optical energy is stored in the low-loss silicon rather than high-loss III-V materials [18, 19], leading to a significant increment of the intrinsic quality factor and decrement of laser gain compared to the conventional III-V DFB lasers, which allows us to use high-Q resonators with high-reflectivity mirrors without sacrificing the lasers' output power as in that case, the three parameters in equation (3) of the heterogenous $\mathrm{Si} / \mathrm{III}-\mathrm{V}$ lasers get reduced simultaneously by roughly the same amount relative to conventional III-V DFB lasers. Hence, its wall plug efficiency, which is the ratio between $T_{\text {opt }}$ and $T_{\text {opt }}+L_{i}$, would be comparable to that of the conventional III-V

DFB lasers. However, when the laser internal field becomes very strong so that the nonlinear loss inside the silicon resonator, such as two-photon absorption, cannot be ignored, then equation (3) is not valid anymore and the laser efficiency will drop as the output power increases, which in turn limits the maximum laser output power [20]. To achieve high-power heterogeneous Si/III-V lasers, the laser resonators should be quite large so that the internal optical field is not large enough to induce nonlinear effects.

In our experiments, the output power of the Si/III-V laser is just several $\mathrm{mW}$, where nonlinear effects are negligible. The high-reflectivity mirrors can block a significant amount of optical feedback from entering the laser resonators, which brings the expectation that the Si/III-V lasers should be much more robust against optical feedback than the conventional III-V DFB lasers, which will be validated experimentally in the following sections.

\section{LASER FREQUENCY NOISE PSD}

In this section, we are going investigate the lasers' capability of preserving their phase coherence under optical feedback. The experimental setups are shown Fig. 2(a) and (b), in which the red arrows represent the propagation of the optical feedback. The coherent optical feedback loop is constructed by coupling part of the laser output back into the laser cavity via the optical circulator, emulating mirror reflection. A booster optical amplifier (BOA, COVEGA BOA-6460) and a variable optical attenuator (VOA) are inserted into the optical feedback loop in order to control the feedback level. The power fed back is calibrated using a high-precision photodetector. The ASE noise of the same BOA without input serves as the source of the incoherent optical feedback and is directly injected into the laser cavity. The frequency noise PSD of both the Si/III-V laser and the III-V DFB laser is measured at various feedback levels with the standard quadrature-locking method, where an MZI with an FSR of roughly $1.5 \mathrm{GHz}$ is used as the frequency discriminator, as shown in Fig. 2(c). In the measurement, the laser is locked to its quadrature point with a feedback circuit and the frequency noise PSD can be calculated based on the power spectrum of the detected signal [21].

The coherent optical feedback level is quantified by

$$
\frac{\gamma P_{\text {feedback }}}{P_{\text {out }}}
$$

, where $\gamma$ is the coupling efficiency between the laser and the optical fiber. $P_{\text {feedback }}$ is the power of the coherent optical feedback measured by the photodetector (PD) and $P_{\text {out }}$ is the total laser output power. The incoherent optical feedback level is quantified by

$$
\gamma P_{\text {feedback }}^{\prime}
$$

, where $P_{\text {feedback }}^{\prime}$ is the power of the incoherent optical feedback, which has been previously calibrated. In our measurements, the coupling efficiency of the Si/III-V laser to the optical fiber is roughly $25 \%$ while that number of the conventional III-V DFB laser, as it has been packaged, is unknown and assumed to be $70 \%$, a reasonable number, in the calibration process.
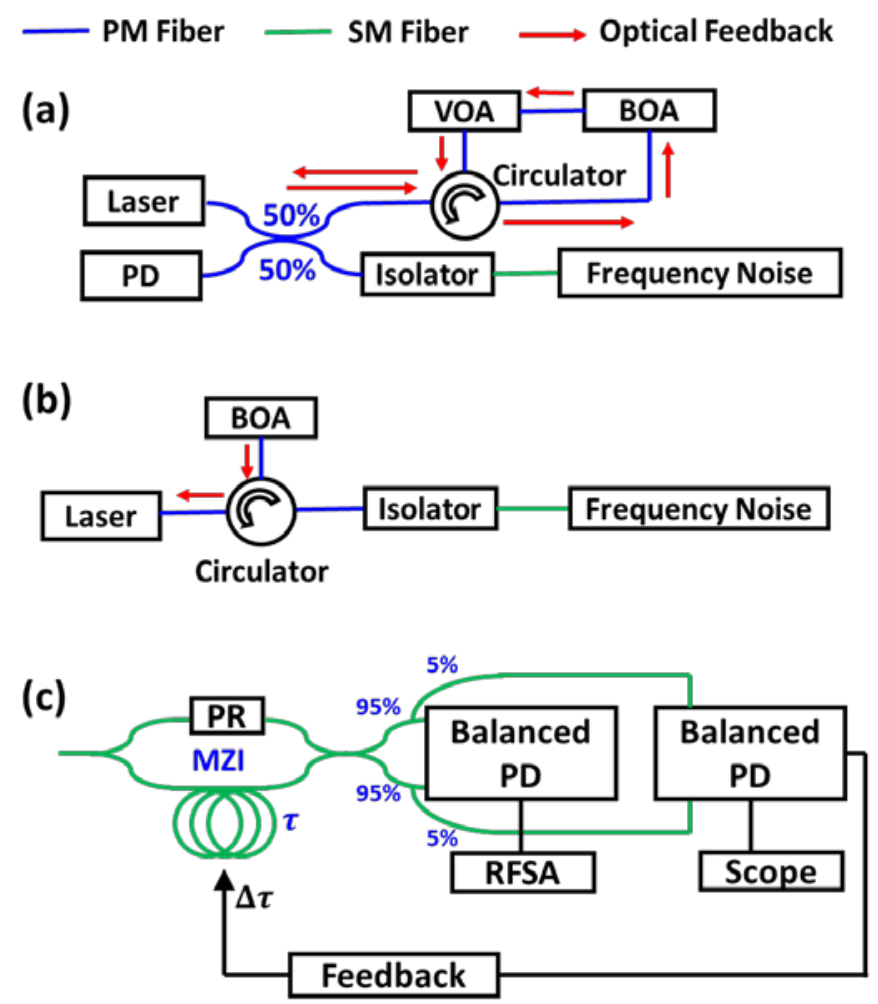

Fig. 2. Measurement setup for (a) coherent optical feedback sensitivity, (b) incoherent optical feedback sensitivity and (c) laser frequency noise PSD. VOA: variable optical attenuator; BOA: booster optical amplifier; PM: polarization maintaining; SM: single mode. PR: polarization rotator. RFSA: radio-frequency spectrum analyzer. PD: photodetector.

The results on lasers' sensitivity to coherent optical feedback are shown in Fig. 3. The III-V DFB laser is very sensitive to the 
feedback as the RF oscillations in the spectrum start to appear at very low feedback level, namely $-50 \mathrm{~dB}$, indicating the onset of coherence collapse. The measurement results do match the original theory [11]. The rising of those side modes results in the loss of laser coherence and eventually the failure of coherent optical communications. On the contrary, the Si/III-V laser is very robust up to the feedback level of $-31 \mathrm{~dB}$, where no significant changes exist in the spectrum. Beyond that, the frequency noise at very low frequencies, i.e. below $100 \mathrm{MHz}$, starts to surge. Such phenomenon, which is completely different from the theory, can be attributed to large thermal effects in the heterogenous Si/III-V lasers [22], which significantly modifies the coupling between laser intensity and phase noise at very low frequencies but is neglected in the original theory because of very small thermal impedance of the conventional III-V DFB lasers. Such an explanation is supported by the fact that the frequency range of the increased frequency noise, i.e. below $100 \mathrm{MHz}$, is the same as the frequency range where the coupling increases sharply because of the thermal effects [23].

The phase noise in the coherent optical communication can be expressed as

$$
\sigma_{\psi}^{2}=4 \int_{0}^{+\infty} S_{\Delta v}(f) \frac{\sin ^{2}\left(\pi f \tau_{0}\right)}{f^{2}} d f
$$

, where $\sigma_{\psi}$ is the standard variance of the phase noise in the coherent communication system, $\tau_{0}$ is the time difference between two successive symbols and $S_{\Delta v}(f)$ is the frequency noise PSD of the laser carrying the information. The increase of the frequency noise at relatively low frequency won't jeopardize the system performance severely because of its small bandwidth and therefore very limited contribution to the phase noise. From a different perspective, such an effect only results in a slow drift of the lasing frequency, which can be easily compensated in the digital signal processing.

It is worth mentioning that, unlike the commercial III-V DFB laser, the Si/III-V laser is unpackaged, leading to poor thermal management. We do expect its robustness to be improved with simply better packaging. Based on the previous results, the $\mathrm{Si} / \mathrm{III}-\mathrm{V}$ laser is at least $19 \mathrm{~dB}$ more stable than the conventional III-V DFB laser against coherent optical feedback. (a)

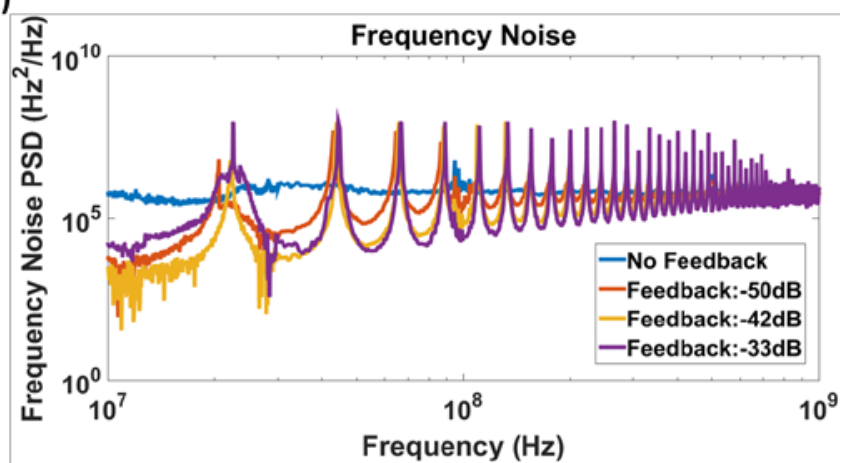

(b)

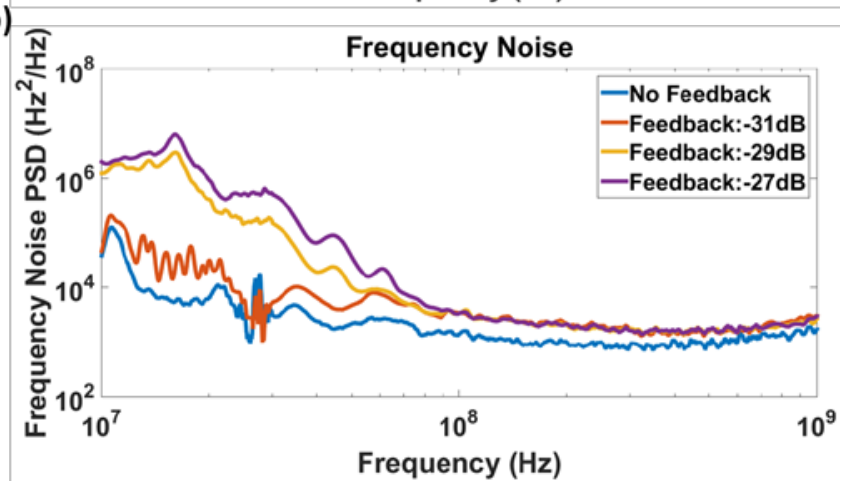

Fig. 3. Frequency noise PSD of (a) III-V DFB laser and (b) Si/III-V laser under coherent optical feedback.

In the case of incoherent optical feedback, the laser frequency noise PSD stays white and increases with the injected ASE power, which is shown in Fig. 4(a). The Schawlow-Townes linewidth, interpreted by the white noise floor [18], i.e. the black dash lines, is plotted as a function of the ASE power fed back in Fig. 4(b). The green curves are linear regression, which fit the data well, indicating the laser frequency noise PSD increases linearly with the ASE power. The huge difference between the high-coherence Si/III-V laser and the conventional III-V DFB laser lies at the slopes of the two curves. The slope extracted for the III-V DFB laser is 32 $\mathrm{MHz} / \mathrm{mW}$ while only $0.2 \mathrm{MHz} / \mathrm{mW}$ for the Si/III-V laser, which is two orders of magnitude smaller.

In summary, we have demonstrated that the Si/III-V laser is more robust than the conventional III-V DFB laser and capable of preserving its phase coherence under much larger optical feedback, making it possible for the Si/III-V laser to function without severe degradation in an isolator-free coherent optical communication system. Such an expectation will be verified in the following sections. 
(a)

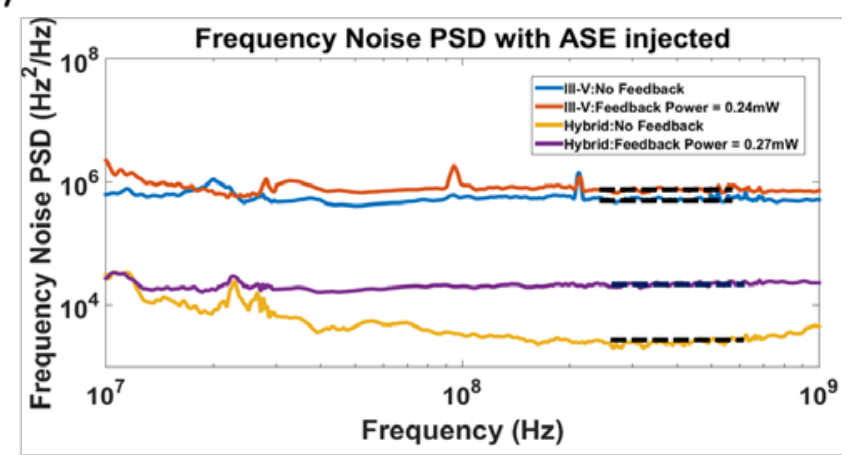

(b)

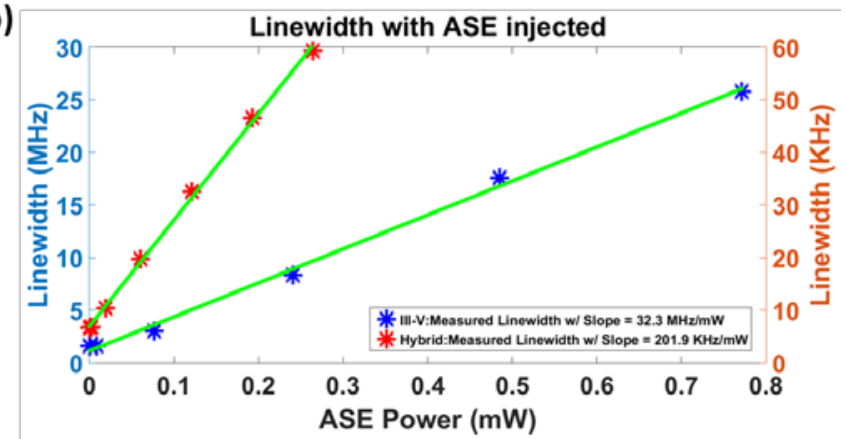

Fig. 4. (a) Frequency noise PSD of the III-V laser and Si/III-V laser under incoherent optical feedback (b) Schawlow-Townes linewidth as a function of ASE power.

\section{MEASUREMENT SETUP FOR COMMUNICATION}

The setup for the performance measurements in a coherent communication system is shown schematically in Fig. 5. The construction of the optical feedback loops is the same as before. Both the Si/III-V laser and the III-V DFB laser are tested for comparison. The output power of both lasers is around $0 \mathrm{dBm}$, which is boosted before the inphase-quadrature (IQ) modulator using an erbium-doped fiber amplifier (EDFA).

The semiconductor lasers, subject to a variable-controlled optical feedback, are used as the light source to carry data signals. At the transmitter side, 20 Gbaud 16 quadrature amplitude modulation (16-QAM) and quadrature phase shift keying (QPSK) signals generated by an arbitrary waveform generator (AWG) at a sampling rate of $92 \mathrm{GSa} / \mathrm{s}$ are modulated onto the Si/III-V laser and III-V DFB laser, respectively. The modulation formats are chosen based on the lasers' intrinsic coherence. The signals are pulse shaped by raise-cosine filters with the roll-off factor of 0.35 . To avoid other transmission effects in the fiber link, back-to-back (BTB) communication is conducted here. A variable optical attenuator (VOA) is used to adjust the optical signal to noise ratio (OSNR). At the receiver side, the signal is pre-amplified with an EDFA and an optical band pass filter with the bandwidth of $1 \mathrm{~nm}$ is placed in front of the coherent receiver to suppress the out-of-band noise. The sampled signals are then processed offline. In the digital signal processing (DSP) module, phase recovery is realized using the algorithm based on Kalman filter throughout all the experiments [24]. Eventually, bit error rate (BER) is measured as a function of the OSNR at various feedback levels. The shift of the BER-OSNR curve would be an indication of the degradation due to optical feedback.

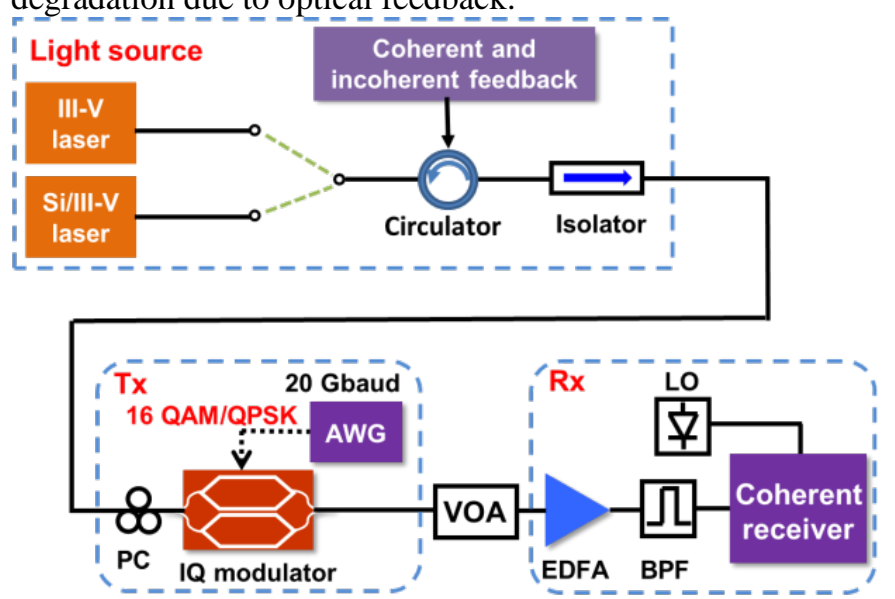

Fig. 5. Experimental setup for evaluating the performance of the III-V DFB laser and the Si/III-V laser in the coherent optical communication. EDFA: erbium-doped fiber amplifier; Tx: transmitter; Rx: receiver; AWG: arbitrary waveform generator; VOA: variable optical attenuator; PC: polarization controller; BPF: band-pass filter; LO: local oscillator.

\section{SySTEM PERFORMANCE}

The lasers' system performance under coherent optical feedback is shown in Fig. 6. The Si/III-V laser is quite robust as there are no significant changes of the BER-OSNR curves even at feedback level up to $-18.3 \mathrm{~dB}$, the largest feedback level employed in the experiments. In contrast, in the case of the III-V DFB laser, the BER-OSNR curve starts to shift upwards at the feedback level of $-45.5 \mathrm{~dB}$. Beyond $-41.5 \mathrm{~dB}$, the communication system is driven into chaos and the BER increases significantly due to the increase of the side modes. In this case, the phase information encoded in the III-V DFB laser is completely washed out, as indicated by the constellation diagrams in Fig. 7 (a) and (b).

Based on the data, the Si/III-V laser is more robust against coherent optical feedback than the conventional III-V DFB laser by at least $27.2 \mathrm{~dB}$. For the record, commercial optical isolators typically provide optical isolation between $25 \mathrm{~dB}$ and $30 \mathrm{~dB}$, which suggests that, in terms of sensitivity to coherent optical feedback, the $\mathrm{Si} / \mathrm{III}-\mathrm{V}$ laser is as stable as the commercial III-V DFB laser packaged with an optical isolator.

On the other hand, as shown in Fig. 8, the BER-OSNR curves of the Si/III-V laser are also barely affected by incoherent optical feedback. However, those of the III-V DFB laser keep shifting upwards with the increase of the feedback power, showing the degradation of its system performance. The constellation diagram in Fig. 7 (c) illustrates the large increase of the phase noise in the system, which agrees with the experimental results in section III.

Throughout all the experiments, the constellation diagrams of the heterogenous Si/III-V laser stay almost unchanged, of which a typical one is shown in Fig. 7 (d). It shows that the phase noise in the 16-QAM communication system under optical feedback remains to be small, which again agrees with the experimental results in section III. 

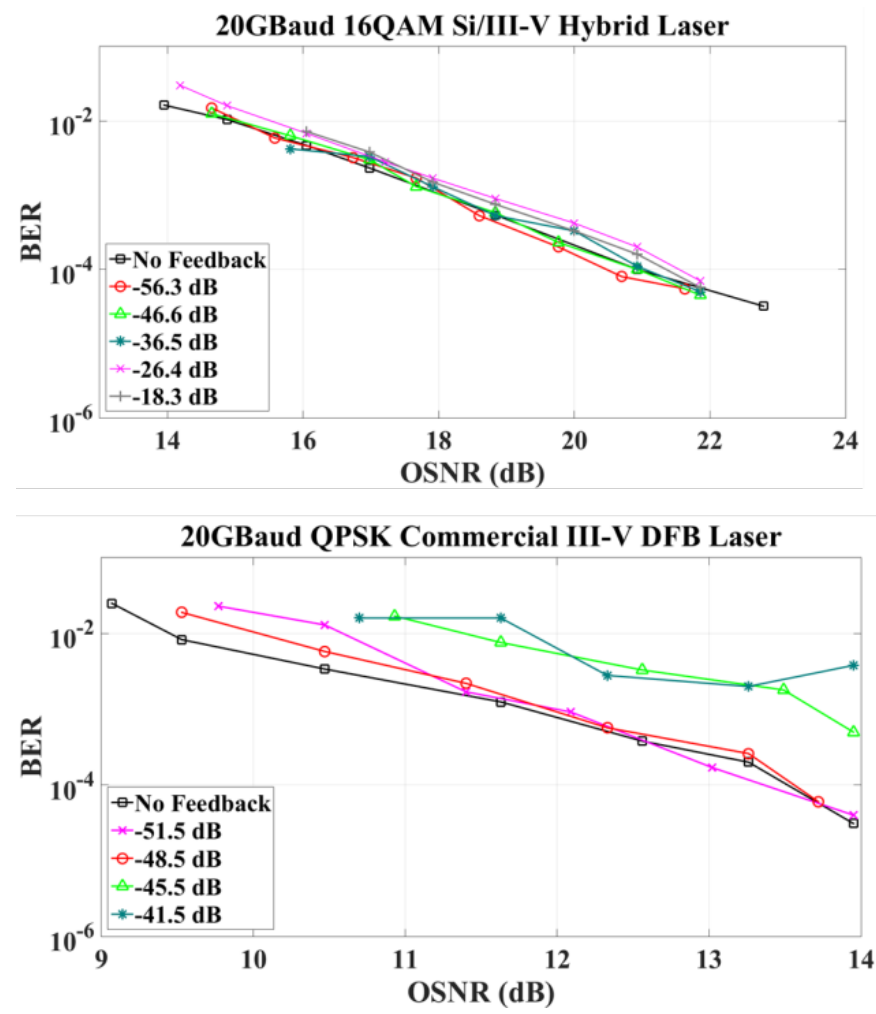

Fig. 6. Performance of the Si/III-V laser and the III-V DFB laser under coherent optical feedback in the coherent optical communication system.
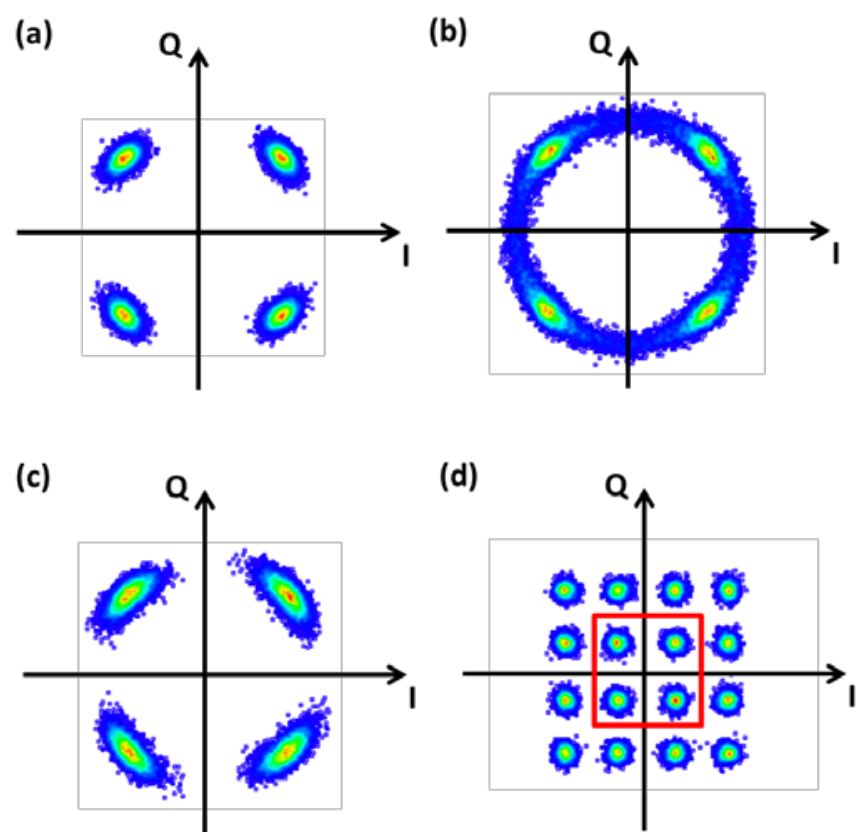

Fig. 7. QPSK constellation diagrams of the III-V DFB laser (a) without coherent or incoherent feedback, (b) with coherent feedback beyond -41dB, (c) with incoherent feedback at $0.7 \mathrm{~mW}$ and (d) a typical 16-QAM constellation diagram of the Si/III-V laser under optical feedback. The color represents the probability distribution of decoded data.
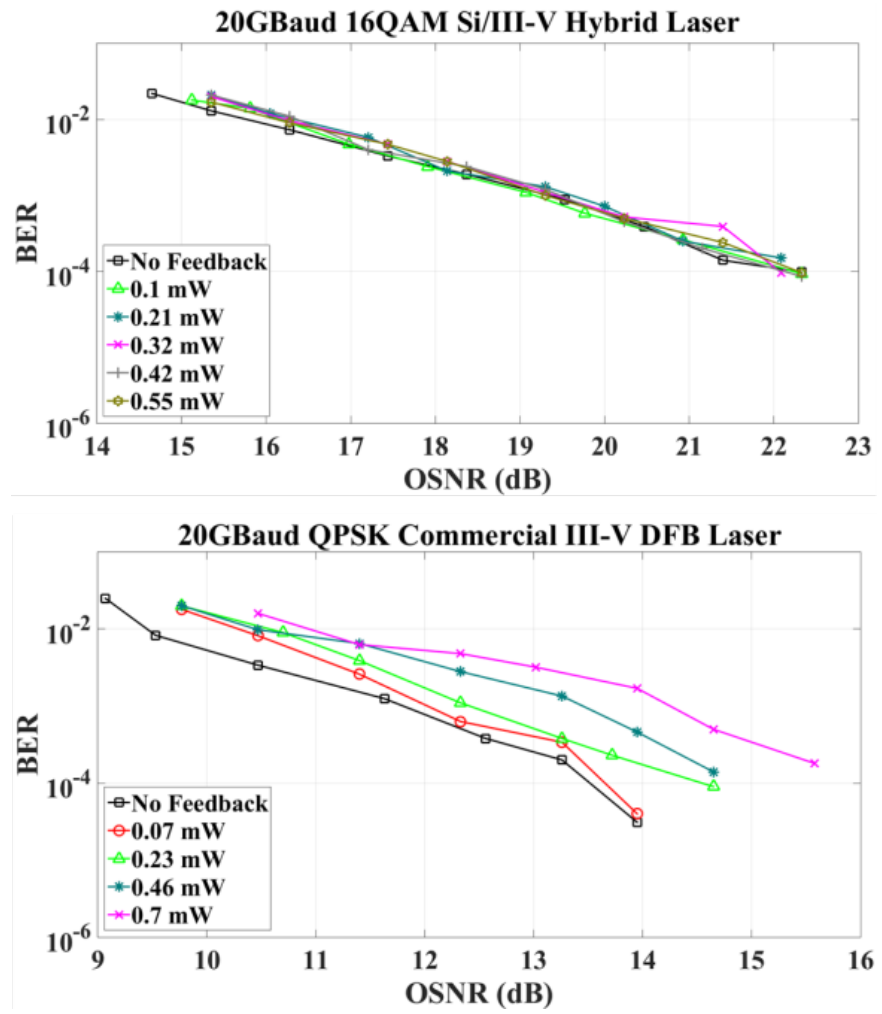

Fig. 8. Performance of the Si/III-V laser and III-V DFB laser under incoherent optical feedback in the coherent optical communication system.

To quantify the feedback effects in a more straightforward way, the OSNR penalty at the BER of $10^{-3}$ is shown in Fig. 9. The penalty for the Si/III-V laser is less than $1 \mathrm{~dB}$ in all cases, showing its robustness against both coherent and incoherent optical feedback. While for the conventional III-V DFB laser, coherent optical feedback at $-46.6 \mathrm{~dB}$ can cause an OSNR penalty of $2 \mathrm{~dB}$. The OSNR penalty approaches infinity at the feedback level beyond $-41.5 \mathrm{~dB}$ because of the chaotic system performance. Besides, incoherent optical feedback can cause a maximum OSNR penalty of $2.5 \mathrm{~dB}$.

It is worth mentioning that 16-QAM is less tolerant to phase noise than QPSK, which strengthens the fact that the Si/III-V laser is much more capable of preserving their phase coherence under optical feedback than the conventional III-V DFB laser. It is expected that the feedback-induced power penalty of the Si/III-V laser for QPSK would be even smaller [25]. From a different perspective, as QPSK is essentially a subset of 16-QAM, indicated by the red box in Fig. 7 (d), where there is no sign of large phase noise, the Si/III-V laser can, without doubt, function properly under optical feedback for QPSK. Our results show that the Si/III-V laser can indeed function without severe degradation in the isolator-free coherent optical communication system, a precondition to its inclusion in photonic integrated circuits. 

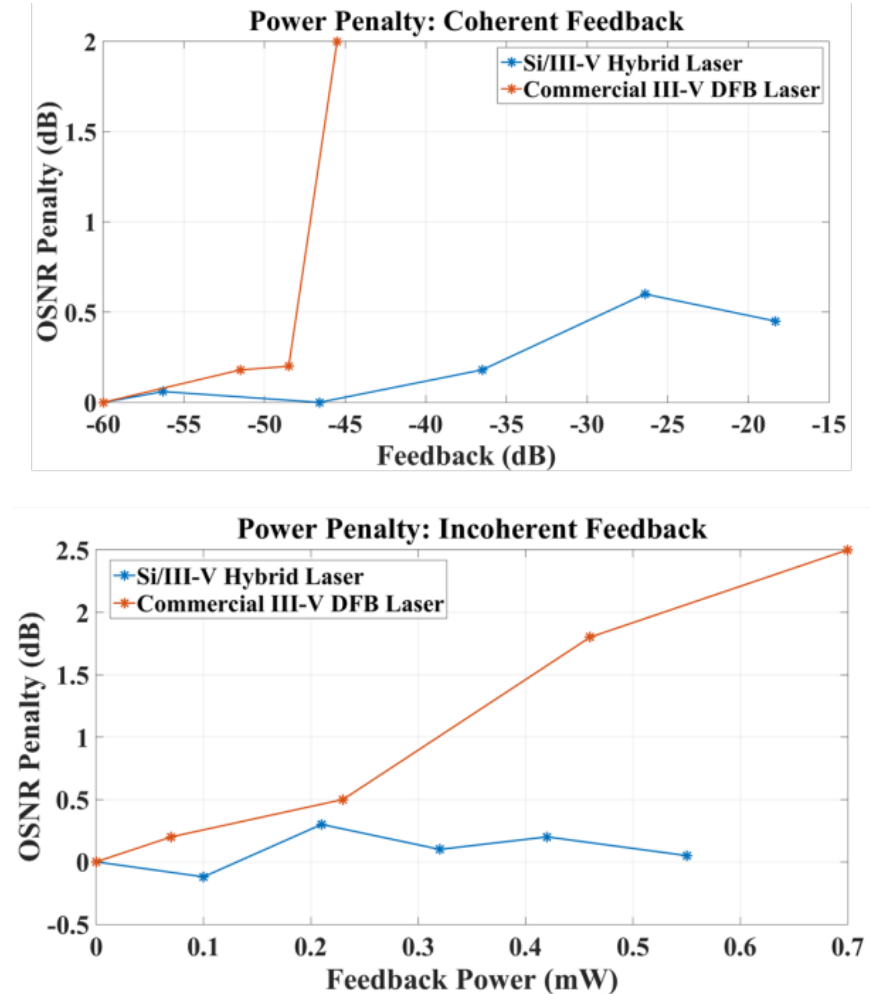

Fig. 9. OSNR penalty at the BER of $10^{\wedge}-3$ due to coherent and incoherent feedback, respectively.

\section{CONCLUSIONS}

To reduce the cost of the emerging coherent optical communication systems, we propose to use the semiconductor lasers with intrinsic insensitivity to optical feedback as the light sources on the photonic integrated platforms. The Si/III-V laser, with its built-in high-Q resonator, has been identified to be a promising candidate. Its robustness and superiority to conventional III-V DFB lasers are confirmed experimentally. More importantly, it can function properly in the isolator-free coherent optical communication system.

\section{REFERENCES}

[1] F. Buchali, M. Chagnon, and K. Schuh, "Transmission Link Optimization for Coherent $4 \mathrm{~Tb} / \mathrm{s}$ Extended Reach (ZR) Transmission," in Optical Fiber Communication Conference (OFC) 2019, San Diego, California, 2019, p. W2A.32.

[2] K. Zou, Z. Zhang, P. Liao, H. Wang, Y. Cao, A. Almaiman, et al., "Using a Hybrid Si/III-V Semiconductor Laser to Carry 16- and 64-QAM Data Signals over an 80-km Distance," in Optical Fiber Communication Conference (OFC) 2019, San Diego, California, 2019, p. M3A.2.

[3] E. Maniloff, S. Gareau, and M. Moyer, "400G and Beyond: Coherent Evolution to High-Capacity Inter Data Center Links," in Optical Fiber Communication Conference (OFC) 2019, San Diego, California, 2019, p. M3H.4.

[4] M. Luo, D. Wu, W. Li, T. Zeng, L. Zhou, L. Meng, et al., "Demonstration of Bidirectional Real-Time $100 \mathrm{~Gb} / \mathrm{s}(4 \times 25 \mathrm{~Gb} / \mathrm{s})$ Coherent UDWDM-PON with Power Budget of $44 \mathrm{~dB}$," in Optical Fiber Communication Conference (OFC) 2019, San Diego, California, 2019, p. Th3F.2.

[5] U. Fiedler and K. J. Ebeling, "Design of VCSEL's for feedback insensitive data transmission and external cavity active mode-locking," IEEE Journal of Selected Topics in Quantum Electronics, vol. 1, pp. 442-450, 1995.

[6] R. Tkach and A. Chraplyvy, "Regimes of feedback effects in 1.5- $\mu \mathrm{m}$ distributed feedback lasers," Journal of Lightwave Technology, vol. 4, pp. 1655-1661, 1986.

[7] J. Duan, H. Huang, B. Dong, D. Jung, J. C. Norman, J. E. Bowers, et al., "1.3- um Reflection Insensitive InAs/GaAs Quantum Dot Lasers Directly Grown on Silicon," IEEE Photonics Technology Letters, vol. 31, pp. 345-348, 2019.

[8] J. Duan, H. Huang, B. Dong, J. C. Norman, Z. Zhang, J. E. Bowers, et al., "Dynamic and nonlinear properties of epitaxial quantum dot lasers on silicon for isolator-free integration," Photonics Research, vol. 7, pp. 1222-1228, 2019/11/01 2019.

[9] H. Huang, J. Duan, B. Dong, J. Norman, D. Jung, J. E. Bowers, et al., "Epitaxial quantum dot lasers on silicon with high thermal stability and strong resistance to optical feedback," APL Photonics, vol. 5, p. 016103, 2020/01/01 2020.

[10] S. Gomez, H. Huang, J. Duan, S. Combrié, A. Shen, G. Baili, et al., "High coherence collapse of a hybrid III-V/Si semiconductor laser with a large quality factor," Journal of Physics: Photonics, vol. 2, p. 025005, 2020/04/22 2020.

[11] C. Henry, "Theory of the linewidth of semiconductor lasers," IEEE Journal of Quantum Electronics, vol. 18, pp. 259-264, 1982.

[12] N. Schunk and K. Petermann, "Minimum bit rate of DPSK transmission for semiconductor laser with a long external cavity and strong linewidth reduction," Journal of Lightwave Technology, vol. 5, pp. 1309-1314, 1987.

[13] Z. Zhang and A. Yariv, "A General Relation Between Frequency Noise and Lineshape of Laser Light," IEEE Journal of Quantum Electronics, vol. 56, pp. 1-5, 2020.

[14] H. Li, J. Ye, and J. G. McInerney, "Detailed analysis of coherence collapse in semiconductor lasers," IEEE Journal of Quantum Electronics, vol. 29, pp. 2421-2432, 1993.

[15] K. Petermann, Laser diode modulation and noise vol. 3: Springer Science \& Business Media, 2012.

[16] F. Grillot, B. Thedrez, and D. Guang-Hua, "Feedback sensitivity and coherence collapse threshold of semiconductor DFB lasers with complex structures," IEEE Journal of Quantum Electronics, vol. 40, pp. 231-240, 2004.

[17] A. Yariv, Quantum electronics. New York: John Wiley \& Sons, 1989.

[18] C. T. Santis, S. T. Steger, Y. Vilenchik, A. Vasilyev, and A. Yariv, "High-coherence semiconductor lasers based on integral high-Q resonators in hybrid Si/III-V platforms," Proceedings of the National Academy of Sciences, vol. 111, p. 2879, 2014.

[19] C. T. Santis, Y. Vilenchik, N. Satyan, G. Rakuljic, and A. Yariv, "Quantum control of phase fluctuations in semiconductor lasers," Proceedings of the National Academy of Sciences, vol. 115, p. E7896, 2018.

[20] V. Yaakov, T. S. Christos, T. S. Scott, S. Naresh, and Y. Amnon, "Theory and observation on non-linear effects limiting the coherence properties of high-Q hybrid Si/III-V lasers," in Proc.SPIE, 2015.

[21] H. Wang, D. Kim, M. Harfouche, C. T. Santis, N. Satyan, G. Rakuljic, et al., "Narrow-Linewidth Oxide-Confined Heterogeneously Integrated Si/III-V Semiconductor Lasers," IEEE Photonics Technology Letters, vol. 29, pp. 2199-2202, 2017.

[22] M. N. Sysak, D. Liang, R. Jones, G. Kurczveil, M. Piels, M. Fiorentino, et al., "Hybrid Silicon Laser Technology: A Thermal Perspective," IEEE Journal of Selected Topics in Quantum Electronics, vol. 17, pp. 1490-1498, 2011.

[23] D. Kim, M. Harfouche, H. Wang, N. Satyan, G. Rakuljic, and A. Yariv, "Suppression of linewidth enhancement factor in high-coherence heterogeneously integrated silicon/III-V lasers," in 2017 Conference on Lasers and Electro-Optics (CLEO), 2017, pp. $1-2$.

[24] T. Inoue and S. Namiki, "Carrier recovery for M-QAM signals based on a block estimation process with Kalman filter," Optics Express, vol. 22, pp. 15376-15387, 2014/06/30 2014

[25] T. Pfau, S. Hoffmann, and R. Noé, "Hardware-Efficient Coherent Digital Receiver Concept With Feedforward Carrier Recovery for M-QAM Constellations," Journal of Lightwave Technology, vol. 27, pp. 989-999, 2009/04/15 2009. 